\title{
Creación de una metodología de proyectos en Tecnologías de Información y Comunicación en la Universidad Estatal a Distancia
}

\author{
Marco A. Chaves Ledezma* \\ (iD) https://orcid.org/0000-0003-1532-9709
}

Recibido: 20 de agosto, 2019 - Aceptado: 24 de setiembre, 2019

\begin{abstract}
RESUMEN
Este trabajo resume la ruta tomada por la Universidad Estatal a Distancia (UNED) con el fin de que cuente con una metodología de proyectos en Tecnologías de Información y Comunicación (TIC), esto en cumplimiento al mandato de la Contraloría General de la República de Costa Rica (2007)1 ${ }^{1}$, el cual en el punto 1.5 dicta: «la organización debe administrar sus proyectos de TI de manera que logre sus objetivos, satisfaga los requerimientos y cumpla con los términos de calidad, tiempo y presupuesto óptimos preestablecidos».

Con el fin de crear esta metodología, se emprendió la tarea de diseñarla a partir de una revisión de literatura de las metodologías PMBOK ${ }^{\circledR}$, SCRUM, ÁGIL, PRINCE2 ${ }^{\circledR}$ y la realización de una pasantía en la Universidad Técnica Federico Santa María (UTFSM), en Chile.

La aplicación de la metodología de proyectos TIC permite a las instituciones el uso de buenas prácticas, comprobadas internacionalmente, que aseguren su éxito.
\end{abstract}

Palabras clave: Proyectos en TIC, metodología de desarrollo, gestión, riesgo.

\section{Formato de citación según APA}

Chaves-Ledezma, M. (2020). Creación de una metodología de proyectos en Tecnologías de Información y Comunicación en la Universidad Estatal a Distancia. Revista Espiga, 19 (39), 27-45.

\section{Formato de citación según Chicago-Deusto}

Chaves-Ledezma, Marco A. (2020). «Creación de una metodología de proyectos en Tecnologías de Información y Comunicación en la Universidad Estatal a Distancia». Revista Espiga 19, n. ${ }^{\circ} 39$ (enero-junio, 2020): 27-45.

* Magíster en Administración de la Tecnología de la Información con énfasis en Administración de Proyectos Informáticos de la Universidad Nacional (UNA), de Costa Rica. Bachillerato en Informática Administrativa de la Universidad Estatal a Distancia (UNED), de Costa Rica. Profesor e investigador de la Cátedra de Tecnologías de la Información de las carreras de Bibliotecología de la Escuela de Ciencias Sociales y Humanidades (UNED) y Coordinador de Proyectos en TIC de la Vicerrectoría de Planificación de la UNED, de Costa Rica. Correo: mchavesl@uned.ac.cr

1. Normas técnicas para la gestión y el control de las Tecnologías de Información N-2-2007-CODFOE, Contraloría General de la República $(5,2007)$. 


\section{Marco contextual}

La Universidad Estatal a Distancia (UNED), de Costa Rica, fue creada en 1977. En América, se distingue por ser una de las universidades concebidas, desde el inicio, con una modalidad a distancia, lo cual ha generado que se conciban estructuras y flujos de trabajo propios, con el fin de responder al modelo y a las necesidades operativas.

Para el 2012, la Contraloría General de la República (CGR) reafirma, para las universidades del sector público, el cumplimiento de las Normas Técnicas para la Gestión y Control de Tecnologías de Información: N-2-2007-CO-DFOE ${ }^{2}$, dentro de las que se encuentra la Norma 1.5, que se refiere a la creación de una metodología de proyectos. La norma dicta: «La organización debe administrar sus proyectos de TI de manera que logre sus objetivos, satisfaga los requerimientos y cumpla con los términos de calidad, tiempo y presupuesto óptimos preestablecidos». Con el fin de cumplir con esta norma, la UNED toma dos decisiones: 1. crear una Coordinación de Proyectos en TIC y 2. crear la Comisión Estratégica de Ciencia y Tecnología (CETIC).

\section{Creación de la Coordinación de Proyectos}

En el 2014, la Vicerrectoría de Planificación, en la persona del Dr. Edgar Castro Monge, vicerrector a cargo, se da a la tarea de cumplir con lo establecido por la CGR e inicia la gestión ante la administración de la universidad con el fin de crear la Coordinación de Proyectos en TIC. Estos esfuerzos fueron plasmados en el acuerdo (perfil definido por la Oficina de Recursos Humanos de la UNED, 2014), en el cual se crea esta coordinación y se nombra al autor de este artículo como su coordinador.

La Coordinación de Proyectos en TIC tiene como objetivo general: «Crear la metodología de proyectos TIC y otros documentos que permita a las diferentes dependencias de la universidad: planificar, coordinar, dirigir, supervisar y evaluar todas las actividades, técnicas y administrativas correspondientes a los proyectos en el área de Tecnologías de Información y Comunicación» ${ }^{3}$. Para lograrlo, esta oficina debe colaborar, coordinar y articular con los responsables de proyectos de TIC en la definición de acciones correctivas; dar seguimiento y proponer mejoras de control en proyectos relacionados con TIC; asesorar a funcionarios, grupos de trabajo e instancias en la definición y sistematización de los procesos, procedimientos y organización relacionados con las TIC; asesorar a las diferentes comisiones relacionadas con proyectos TIC en la definición y ejecución de procedimientos de control de cambios para asegurar el plan de continuidad de TIC ${ }^{4}$.

Dentro de sus funciones se enumeran las siguientes:

- Planear, coordinar, supervisar y dirigir las actividades correspondientes a su ámbito de acción.

\footnotetext{
2. Ibíd.

3. Perfil definido por la Oficina de Recursos Humanos de la UNED, 2014.

4. Ibíd.
} 
- Asesorar a funcionarios, grupos de trabajo e instancia en la definición y sistematización de los procesos, procedimientos y organización relacionados con las TIC.

- Asesorar a las comisiones (Comisión Estratégica de Tecnologías de Información, CETIC) y todas aquellas en materia TIC.

- Asesorar en materia de inversión en TIC a las comisiones en TIC (definición de prioridades, elaboración de presupuestos, administración de costos, evaluaciones de los beneficios de los proyectos).

- Asesorar en la definición y administración de los acuerdos de niveles de servicio a toda la función.

- Canalizar la definición de los requerimientos técnicos que definen las comisiones institucionales de TIC.

- Ser el canal de comunicación entre las comisiones institucionales (las que existen en la UNED) y viceversa, y entre las áreas usuarias y estos órganos.

- Monitorear el estado de los proyectos de TIC y reportar sus desviaciones.

- Dar seguimiento y proponer mejoras de control en proyectos relacionados con TIC.

- Elaborar indicadores que permitan la generación de informes para el monitoreo del inicio, ejecución y cierre de los proyectos de TIC.

- Colaborar, coordinar y articular con los responsables de proyectos de TIC en la definición de acciones correctivas.

- Desarrollar métricas que permitan la medición de los niveles de servicio en materia de TIC, así como los planes de mejora continua.

- Velar para que los proyectos elaborados cumplan con la aplicación de la normativa institucional y nacional vigente, relacionada con TIC.

- Divulgar información relativa a los procesos y procedimientos en TIC a la comunidad universitaria, por medio de la publicación de artículos técnicos y profesionales, charlas y conferencias.

- Poner en práctica las resoluciones o acuerdos del Consejo Universitario, del Consejo de Rectoría y de las comisiones institucionales en TIC, relacionados con su campo de acción.

- Velar por la correcta utilización de los materiales, activos, equipos y otros valores asignados para el ejercicio de su función.

- Hacer evaluaciones periódicas de los resultados de la aplicación y desarrollo de las políticas, planes y programas bajo su responsabilidad y efectuar los cambios y ajustes necesarios para el logro de los objetivos establecidos.

- Elaborar y presentar los informes de labores correspondientes a su ámbito de acción.

- Participar en todas las comisiones institucionales que tengan relación con las TIC. 
- Dar seguimiento a la aplicación de las Normas TIC de la CGR, mediante el grupo de trabajo que se cree.

- Participar en la construcción de los planes TIC a nivel institucional y dar seguimiento a su aplicación.

- Coordinar y colaborar con la instancia de Control Interno en la confección y aplicación de controles en la institución ${ }^{5}$.

\section{Creación de la CETIC}

Otro de los lineamientos, según se lee en la Norma $1.6^{6}$, establece que:

«El jerarca debe apoyar sus decisiones sobre asuntos estratégicos de TI en la asesoría e incluir y conformar una comisión estratégica en Tecnologías de Información que coadyuve a mantener la concordancia con la estrategia institucional, a establecer las prioridades de los proyectos de TI, a lograr un equilibrio en la asignación de recursos y a la adecuada atención de los requerimientos de todas las unidades de la organización».

Con el fin de cumplir esta norma, en el 2014, la administración de la universidad decide crear la Comisión de Ciencia y Tecnología y la Comisión Estratégica de Tecnologías de Información y Comunicación (CETIC), integrada de la siguiente manera:

- $\quad$ Rector (quien preside).

- Vicerrector de Planificación.

- Vicerrector Académico.

- Vicerrector de Investigación.

- Vicerrector Ejecutivo.

- Director de la Dirección de Tecnología, Información y Comunicaciones (DTIC), para brindar la perspectiva técnica de acuerdo con las prioridades en materia de tecnología de información.

- Director Financiero, para asesorar al comité en materia de recursos financieros institucionales y así asegurar el contenido presupuestario de los proyectos.

- Jefe del Centro de Programación y Planificación Institucional (CPPI), para garantizar que los proyectos aprobados por la CETIC estén alineados con los Planes de Desarrollo, Estratégicos y el Plan Operativo Anual.

- Coordinador del Programa de Valoración de la Gestión Administrativa y el Riesgo Institucional (PROVAGARI), para asegurar el cumplimiento de normativa técnica, control interno y el aseguramiento de la mitigación de los riesgos institucionales.

5. Ibíd.

6. Normas técnicas... $(5,2007)$. 
- Coordinador del Programa de Gobierno Digital, que se convierte en figura importante para toda la organización, ya que responde a la estrategia de desarrollo propia de la UNED y a sus lineamientos de política institucional.

- Coordinador de proyectos en TIC (invitado permanente), que, entre algunas de sus funciones, tendrá que colaborar y buscar la articulación y vinculación con todos los proyectos institucionales relacionados en materia de $\mathrm{TIC}^{7}$.

En el acuerdo de creación de la CETIC también es posible discriminar las obligaciones que cada uno de sus miembros asume:

- Ser funcionario con el liderazgo y autoridad requeridos para guiar los proyectos en TIC de la universidad.

- Conocer acerca de la aplicación de metodologías de proyectos en TIC.

- Decidir o resolver en su campo de especialidad o competencia de manera tal que representen el sector por el cual fueron llamados a esta comisión.

- Conocer y estar comprometido con la misión y visión de la universidad.

- Ser participantes activos y comprometidos con esta comisión ${ }^{8}$.

De esta resolución, se desprende que la CETIC debe contribuir en la gestión de proyectos en materia de TIC y asegurar que estos, a su vez, responden a los Lineamientos de Política Institucional 2007-2011 y al Plan de Desarrollo Institucional: para el fortalecimiento de la Educación a Distancia 2011-2015, ambos aprobados por el Consejo Universitario en el 2011. Asimismo, si algún proyecto está incluido en el Acuerdo de Mejoramiento Institucional (AMI), financiado por el Banco Mundial, igualmente debe indicarse a cuál proyecto responde del Plan de Mejoramiento Institucional (PMI).

Es importante señalar que en el diseño e implementación de la metodología de proyectos para la universidad, la CETIC tiene como función el aprobar la propuesta que la Coordinación de Proyectos y la Vicerrectoría de Planificación presenten, así como generar las condiciones propicias para su implementación.

\section{Metodología de la experiencia}

Para cumplir con la tarea encomendada a la Coordinación de Proyectos en TIC, por la administración de la universidad, como metodología de trabajo se definió, primeramente, realizar una revisión de literatura que permitiera reconocer las mejores prácticas en materia de desarrollo de proyectos en TIC, de manera que se pudiera identificar aquella que respondía a las necesidades institucionales. Para esto, se revisaron las siguientes metodologías de proyectos en TIC: PMBOK ${ }^{\circledR}$, SCRUM, ÁGIL y PRINCE2 ${ }^{\circledR}$. La búsqueda se realizó en la base de datos Business Source Complete, de EBSCO, donde se

7. Consejo de Rectoría, 2014-500, Sesión 1824-2014, artículo VI, inciso 1 (21 de julio, 2014).

8. Ibíd. 
revisaron artículos de revistas arbitradas; igualmente, algunos libros especializados en el tema. Se construyó un cuadro comparativo y se realizó un análisis.

En una segunda etapa, y en cumplimiento con la iniciativa 6 del Acuerdo de Mejoramiento Institucional (AMI) ${ }^{9}$, se coordinó y planificó una pasantía en la Universidad Técnica Federico Santa María (UTFSM), ubicada en la ciudad de Valparaíso, Chile. La selección de esta universidad se hizo dado que su especialidad son las ingenierías y, de manera particular, presenta una metodología de proyectos que se consideró necesario revisar.

Como última etapa, se procedió a elaborar el documento de metodología de proyectos para la UNED. En esta etapa se contó con la colaboración de la Dirección de Tecnología de Información y Comunicación (DTIC), el Centro de Investigación y Evaluación Institucional (CIEI) y el Programa de Control Interno (PROCI).

\section{Resultados de la revisión bibliográfica}

La finalidad de la guía del $\mathrm{PMBOK}^{\circledR 10}$ es identificar una serie de componentes en la dirección de los proyectos que generalmente son llamadas buenas prácticas. De esta manera, la organización que utilice esta herramienta puede adaptar a su desarrollo de software lo que es más apropiado, dependiendo de cada proyecto en particular, esto lo va a determinar el responsable del equipo de dirección.

Según el Project Management Institute, el $\mathrm{PMBOK}^{\circledR}$ no es una metodología por sí mismo, sino un subconjunto de herramientas, habilidades y conocimientos que deben poseer los desarrolladores para la aplicación de las mejores buenas prácticas, sin que esto signifique que estas siempre van a ser apropiadas para todos los proyectos.

Esta guía metodológica está orientada hacia una gestión predictiva de los proyectos, lo que quiere decir que antes del inicio de cada uno, se debe conocer con anterioridad aspectos relacionados con el producto, servicio o, bien, qué se va a obtener en determinado proyecto, con el propósito de minimizar los riesgos.

Dentro de los principales beneficios señalados por el Project Management Institute en el uso del $\mathrm{PMBOK}^{\circledR}$, se mencionan que: puede ser aplicado a cualquier tipo de proyecto y se le pueden integrar otras metodologías que ayuden a concretar los objetivos; también, está orientado a procesos, es adaptable, es multi-empresa, es decir, habla en el mismo idioma de desarrollo que muchas otras organizaciones fuera de la institución para interactuar con los proyectos.

9. El Acuerdo de Mejoramiento Institucional tiene como «propósito especificar los compromisos que la UNED ha adquirido para la ejecución del financiamiento obtenido por el gobierno de Costa Rica» (UNED, 2018, párr.3). Este contempla la iniciativa 6, Formación y capacitación para el fortalecimiento del modelo de educación a distancia, la cual cubre la posibilidad de que los funcionarios realicen pasantías.

10. Project Management Institute, Inc., Guía de los Fundamentos de la Dirección de Proyectos. Pennsylvania: Newtown Square, 2004. 
Por otra parte, como lo indican Özkan y Kucuk ${ }^{11}$ SCRUM es un proceso en el que se aplica, de manera constante y regular, una serie de buenas prácticas para trabajar en equipos colaborando entre sus miembros, para obtener el mejor resultado posible en los proyectos. Para que estas buenas prácticas sean posibles, es necesario contar con equipos altamente productivos.

Esta metodología generalmente es utilizada en organizaciones que requieren obtener resultados lo más rápidos posibles y en las cuales interactúan diferentes dependencias de la institución en conjunto para obtener los productos de software en el tiempo, costo y con la calidad establecida, de acuerdo con lo señalado por Özkan y Kucuk.

Con SCRUM, los requisitos, necesidades y condiciones pueden ser muy cambiantes; por lo tanto, como indica Ionel, esta metodología debe ofrecer la flexibilidad al desarrollador para hacer que estas condiciones sean diferentes, conforme avanza el proyecto y, además, como se mencionó anteriormente, los resultados puedan obtenerse lo más expedito posible.

Según Machado, Pinheiro y Tamanini ${ }^{12}$, así como Özkan y Kucuk ${ }^{13}$, algunos de sus principales beneficios son: entrega mensual o quincenal de resultados, productividad y calidad, alineamiento entre el cliente y el equipo de desarrollo, mantiene al equipo de trabajo altamente motivado.

Según Walczak y Kuchta ${ }^{14}$, las metodologías Ágiles (ÁGIL), que nacieron en la década de 1990, enfrentan un cambio en el desarrollo de software con aquellas que se les denomina de «peso pesado» o muy rígidas. Nacen a raíz de que en muchos desarrollos de proyectos, los involucrados no tienen claro cuáles son los requerimientos y cambian conforme se avanza.

Dentro de las metodologías Ágiles se le da mayor importancia a la participación de los individuos en el desarrollo de los proyectos, más que a preparar un entorno para su desarrollo. Cole y Borzone indican que se considera la participación y colaboración con el cliente, más que elaborar un contrato de servicios, además es más importante realizar los cambios de manera inmediata que seguir un plan preestablecido para su desarrollo.

De acuerdo con Cole y Borzone ${ }^{15}$, se puede decir que las metodologías Ágiles están más orientadas al día a día en la producción del desarrollo de software, que no siguen patrones establecidos ni prácticas rígidas; tampoco es importante la documentación de

11. Özkan, Necmettin e Kucuk, Civian, «A Systematic Approach to Project Related Concepts of Scrum», Review Of International Comparative Management / Management Comparat International, 17, n. ${ }^{\circ} 4$ (2016): 320-334.

12. Machado, Sampaio, Thais Cristina, Pinheiro, Plácido Rogério y Tamanini, Isabelle, «Project management aided by verbal decision analysis approaches: a case study for the selection of the best SCRUM practices», International Transactions In Operational Research, 22 n. ${ }^{\circ} 2$ (2015): 287-312.

13. Ibíd.

14. Walczak, Wojciech y Kuchta, Dorota, «Risks Characteristic of Agile Project Management Methodologies and Responses to Them», Operations Research \& Decisions, 23, n. ${ }^{\circ} 4$ (2013): 75-95.

15. Cole, Gray y Borzone, Brian, «How Agile Methodology Applies to HR Technology Solution Selection», Workforce Solutions Review, 8, n. ${ }^{\circ} 1$ (2017): 4-6. 
los sistemas, ya que da más valor a los resultados inmediatos que a una planificación de desarrollo.

Cuando los proyectos no están claramente definidos, los beneficios que Maruping, Venkatesh y Agarwa ${ }^{16}$ atribuyen a las metodologías Ágiles cualidades como: flexibilidad en el proceso y las definiciones de los productos, realimentación continua con el cliente, interacción constante y la calidad mejorada.

La metodología PRINCE2 ${ }^{\circledR}$ está preparada para adaptarse a los diferentes proyectos que se desarrollan dentro de las organizaciones. Vaníčková ${ }^{17}$ apunta a que es muy flexible, su enfoque está dirigido, entre otros, a la entrega del producto, por esta razón tiene una orientación hacia aquellas organizaciones cuyo principal objetivo está orientado hacia la entrega del producto; sin embargo, esto no quiere decir que no pueda utilizarse en otro tipo de proyectos y organizaciones.

Según Tuczak y Górzna ${ }^{18}$, esta metodología es utilizada por muchas empresas alrededor del mundo y tiene la particularidad de controlar muy bien los recursos y utilizar la gestión de riesgos para minimizar los fracasos. Entre los beneficios que esta metodología aporta, se puede encontrar: es aplicable a todos los tipos de proyectos, administra el enfoque de negocio del proyecto de forma continua y se adapta a los cambios diariamente si se realizan los ajustes necesarios.

\section{Resultados de la pasantía en la UTFSM, Chile}

En el 2015, se hizo una visita a la Universidad Técnica Federico Santa María (UTFSM), localizada en Valparaíso, Chile, con el fin de obtener información, de fuente primaria, acerca de la forma en que lleva a cabo el desarrollo de los proyectos en TIC. La persona de contacto en esta pasantía fue el ingeniero civil y magíster en ingeniería, Luis Fernando Hevia Rodríguez, encargado de comunicaciones.

La UTFSM es una institución privada que cuenta con sedes en Viña del Mar, Vitacura (campus), San Joaquín (campus), Concepción (sede), Rancagua (oficina) y Guayaquil (campus). Esta institución es líder en la formación en el campo ingenieril, de la ciencia y de la tecnología, según se lee en la página de la universidad ${ }^{19}$.

16. Maruping, Likoebe M., Venkatesh, Viswanath y Agarwal, Ritu, «A Control Theory Perspective on Agile Methodology Use and Changing User Requirements», Information Systems Research, 20, n. ${ }^{\circ} 3$ (2009): 377-399.

17. Vaníčková, Radka, «Application of PRINCE2® Project Management Methodology», Studia Commercialia Bratislavensia, 10, n. ${ }^{\circ} 38$ (2017): 227-238.

18. Tuczak, Jacek, y Górzna, Marcelina, «Ocena Skuteczności Metodyki Zarządzania Projektami PRINCE2 ${ }^{\circledR}$ W Administracji Publicznej», Research Papers Of The Wroclaw University Of Economics / Prace Naukowe Uniwersytetu Ekonomicznego We Wroclawiu, 264 (2012): 282-299.

19. Universidad Técnica Federico Santa María, «Historia», Acceso: 22 de enero, 2020. http://www.usm.cl/ universidad/historia/ 
La información aquí consignada se obtuvo mediante conversación personal con los siguientes funcionarios de la UTFSM:

\begin{tabular}{ll}
\multicolumn{1}{c}{ Nombre } & \multicolumn{1}{c}{ Cargo } \\
Miguel Varas Austudillo & $\begin{array}{l}\text { Jefe de Infraestructura y Tecnología, del Departamento } \\
\text { de Informática. Es el encargado de la división de sistemas } \\
\text { dedicado a la Escuela y Facultad de Informática, que } \\
\text { desarrollan todas las aplicaciones en Linux. }\end{array}$ \\
Jefe de Infraestructura y Seguridad \\
Ricardo Méndez & Funcionario del Departamento de Seguridad \\
Edmundo Venegas & Administrador de sistemas, Unidad de Infraestructura y \\
Yonathan Dossow & $\begin{array}{l}\text { Tecnología. Administra las bases de datos de } \\
\text { este departamento. }\end{array}$ \\
Gonzalo Lefian Mujica & $\begin{array}{l}\text { Ingeniero de soporte del Área de Infraestructura y } \\
\text { Tecnología. Brinda soporte técnico a toda la Facultad y }\end{array}$ \\
Escuela de Informática.
\end{tabular}

Fuente: Elaboración propia.

La UTFSM no cuenta formalmente con una unidad constituida de proyectos en TI, según lo expresado por Luis Hevia ${ }^{20}$. Cada una de las dependencias, tanto administrativas como académicas, son las que proponen, a un comité gerencial una vez cada año, los proyectos de acuerdo con sus necesidades.

Este comité gerencial, que viene a ser el homólogo en la UNED de la CETIC, es el que se encarga de evaluar cada proyecto y de verificar su vialidad y su conveniencia de ejecución, así como de asignarle los recursos necesarios y suficientes para que las unidades encargadas de desarrollarlo cuenten con todos los recursos para finalizarlos.

Según lo mencionado por Luis Reinaldo Vallejos y Miguel Varas ${ }^{21}$, no utilizan una única metodología para la elaboración y ejecución de los proyectos, sino que hacen una

20. Luis Hevia (encargado de Comunicaciones de la Universidad UTFSM), en conversación con el autor, 30 de noviembre, 2015.

21. Luis Reinaldo Vallejos (director de la Escuela de Ingeniería Mecánica) y Miguel Varas (jefe de Infraestructura y Tecnología), en conversación con el autor, 01 de diciembre, 2015. 
integración del $\mathrm{PMBOK}^{\circledR}, \mathrm{COBIT}^{\circledR} 4.1$ y otras metodologías para su implementación, convirtiéndose en un híbrido de metodologías para sus proyectos. Todo depende de la naturaleza de cada proyecto, así la unidad ejecutora se encarga de elegir la que más se adapte al proyecto que van a desarrollar.

\section{Procedimientos de la UTFSM}

La UTFSM tiene, dentro de la estructura de la Dirección de Tecnología de Información (DTI), un departamento denominado Unidad de Innovación y Desarrollo, en este se encuentra un jefe de proyectos, quien es el comisionado de llevar a cabo todos los proyectos que se presenten al comité encargado de su aprobación para la puesta en desarrollo. El organigrama de la Dirección de Tecnología se muestra a continuación:

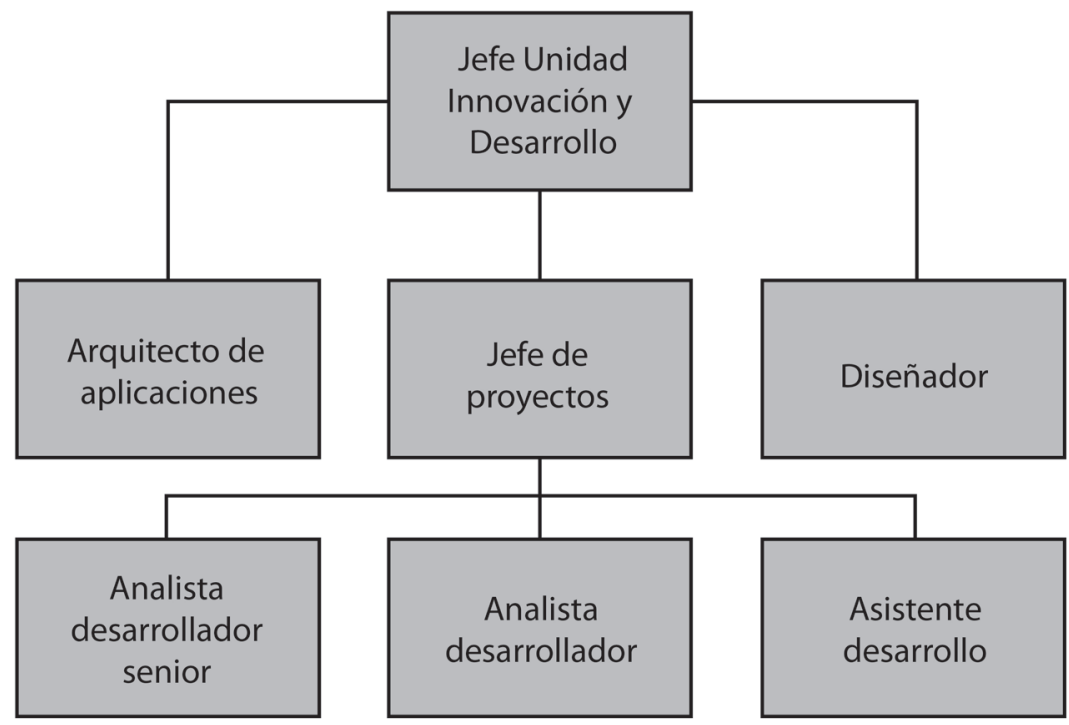

Figura 1. Estructura de la Unidad de Innovación y Desarrollo. Fuente: UTFSM (2015).

Cualquier unidad de la universidad puede y debe desarrollar proyectos de acuerdo con las necesidades que detecten o con los problemas que existan en sus diferentes departamentos.

Con base en lo indicado por Claudia López ${ }^{22}$, una vez que pasan por el comité y son aprobados, cumpliendo con todos los requisitos establecidos, para asegurar su éxito, se trasladan al jefe de proyectos, quien a su vez, y dependiendo de su naturaleza, reúne a sus compañeros de área para establecer los equipos de trabajo y distribuir las funciones y cargas, según las habilidades de cada miembro.

22. López, Claudia, funcionaria-docente del área de Inteligencia computacional aplicada, en conversación con el autor, 02 de diciembre, 2015. 
A partir de la experiencia en la pasantía se pudo constatar que cada unidad, facultad, escuela o dependencia administrativa tiene autonomía en el accionar de sus proyectos y en la escogencia de su infraestructura tecnológica.

En cuanto a hardware y software, cada departamento escoge la configuración de los equipos y los programas que van a instalar de acuerdo con la necesidad particular de la dependencia. No existe un estándar para la adquisición de hardware o software, pero sí cuentan con la asesoría técnica de la DTI para cumplir con esta función.

En sus servidores, la DTI tiene instalados sistemas operativos de Microsoft, pero también algunas oficinas tienen instalado Linux para sus aplicaciones y para la operación.

\section{Innovación para la calidad}

Dentro del organigrama de la Dirección de Tecnología existe una unidad que se llama Innovación y Desarrollo, cuyo objetivo es liderar y conducir todos los proyectos de desarrollo y mejoramiento de los productos y servicios que la DTI entrega a la comunidad.

La UTFSM, por ser una institución orientada en su mayoría a la obtención de profesionales en las áreas ingenieriles, sus proyectos son de aplicación práctica en las empresas, tanto del sector público como privado, en donde presentan sus trabajos y los llevan a la práctica con la supervisión de un grupo de profesores encargado de ser su guía durante el periodo de implementación.

A nivel administrativo, se implementan soluciones que brindan los profesores o personal de apoyo y soporte en cada unidad académica con el fin de innovar a lo interno de los departamentos, al presentar ideas novedosas que ayudan al fortalecimiento de la academia desde la parte docente-administrativa. Para lograr esto, según lo expresado por Luis Hevia y Cecilia Reyes ${ }^{23}$, realizan concursos entre las facultades en los cuales interviene la mayoría del personal y se presentan los proyectos a un comité encargado de evaluarlos. A las ideas más innovadoras y que contribuyen con la administración o la docencia, se les da la oportunidad de ser presentados al Comité Central, a cargo de su aprobación final y de asignarle los recursos para que sea desarrollado e implementado.

\section{Infraestructura tecnológica, comunicaciones y configuración de sistemas}

Esta unidad provee, mantiene y dimensiona hardware, redes y servicios necesarios para el desarrollo, mantención y operación de los sistemas de información, el manejo de la información, políticas de seguridad y respaldos, entre otros aspectos, según Ricardo Méndez y Edmundo Venegas ${ }^{24}$. Una de sus principales funciones es asegurar la continuidad operativa de los servicios tecnológicos de la universidad.

23. Reyes, Claudia, funcionaria de la oficina de Proyectos aplicados a la informática, y Luis Hevia, encargado de Comunicaciones de la UTFSM, en conversación con el autor, 3 de diciembre, 2015.

24. Ricardo Méndez, jefe de Infraestructura y Seguridad, y Edmundo Venegas, funcionario del Departamento de Seguridad, en conversación con el autor, 30 de noviembre, 2015. 
La DTI cuenta con una serie de servidores Linux, algunos virtualizados, otros dedicados a la comunicación, bases de datos, internet y respaldos, entre los más importante. Las demás facultades o dependencias cuentan individualmente y de acuerdo con sus necesidades y requerimientos, con servidores dedicados a sus propósitos. La DTI los instala y brinda mantenimiento, pero son administrados por cada oficina.

\section{Análisis de la experiencia y propuesta}

\section{Análisis de la revisión de literatura}

A continuación, se muestra una tabla comparativa de las cuatro metodologías de desarrollo de proyectos TIC, con el fin de realizar un análisis de cada una de sus principales características.

TABLA 1

Comparación de las metodologías para el desarrollo de proyectos TIC: PMBOK $^{\circledR}$, SCRUM, ÁGIL y PRINCE2 ${ }^{\circledR}$

\begin{tabular}{|c|c|c|c|c|}
\hline Criterios & $\mathrm{PMBOK}^{\circledR}$ & SCRUM & ÁGIL & PRINCE2 ${ }^{\circledR}$ \\
\hline Orientación & $\begin{array}{l}\text { Gestión y desarrollo de } \\
\text { software y procesos }\end{array}$ & $\begin{array}{l}\text { Desarrollo de software } \\
\text { rápido }\end{array}$ & $\begin{array}{l}\text { Desarrollo de } \\
\text { productos }\end{array}$ & $\begin{array}{l}\text { Desarrollo de } \\
\text { software }\end{array}$ \\
\hline $\begin{array}{l}\text { Flexibilidad y } \\
\text { adaptabilidad }\end{array}$ & $\begin{array}{l}\text { Adaptable, pero más } \\
\text { rígida que las demás }\end{array}$ & Adaptable y flexible & Adaptable y flexible & $\begin{array}{l}\text { Adaptable } \\
\text { y flexible }\end{array}$ \\
\hline Aplicabilidad & $\begin{array}{l}\text { Todo tipo de } \\
\text { organización, pero } \\
\text { principalmente aplicado } \\
\text { en empresas de mayor } \\
\text { tamaño }\end{array}$ & Todo tipo de organización & $\begin{array}{l}\text { Todo tipo de } \\
\text { organización }\end{array}$ & $\begin{array}{l}\text { Todo tipo de } \\
\text { organización }\end{array}$ \\
\hline $\begin{array}{l}\text { Nivel de } \\
\text { estructuración }\end{array}$ & Estructurado & No es estructurado & No es estructurado & Estructurado \\
\hline $\begin{array}{l}\text { Principal } \\
\text { beneficio }\end{array}$ & $\begin{array}{l}\text { Multitarea, orientado a } \\
\text { procesos }\end{array}$ & $\begin{array}{l}\text { Mantiene al equipo } \\
\text { de trabajo altamente } \\
\text { motivado, con el fin de } \\
\text { asegurar productividad } \\
\text { y calidad }\end{array}$ & $\begin{array}{l}\text { Resultados rápidos, } \\
\text { interacción constante } \\
\text { con el cliente, la } \\
\text { calidad es un proceso } \\
\text { reiterativo }\end{array}$ & $\begin{array}{l}\text { Controla } \\
\text { recursos } \\
\text { y minimiza } \\
\text { riesgos }\end{array}$ \\
\hline $\begin{array}{l}\text { Plazo de } \\
\text { entrega }\end{array}$ & $\begin{array}{l}\text { A mediano y largo } \\
\text { plazo }\end{array}$ & Mensual o quincenal & Entregas rápidas & $\begin{array}{l}\text { Entregas } \\
\text { rápidas }\end{array}$ \\
\hline $\begin{array}{l}\text { Función del } \\
\text { cliente }\end{array}$ & Menos participativo & $\begin{array}{l}\text { Moderadamente } \\
\text { participativo }\end{array}$ & $\begin{array}{l}\text { Altamente } \\
\text { participativo en el } \\
\text { desarrollo }\end{array}$ & $\begin{array}{l}\text { Muy } \\
\text { participativo }\end{array}$ \\
\hline
\end{tabular}

Fuente: Elaboración propia a partir de Project Management Institute (2004); Özkan y Kucuk (2016); Ionel (2008); Machado, Pinheiro y Tamanini (2015); Walczak y Kuchta (2013); Cole y Borzone (2017); Maruping, Venkatesh y Agarwal (2009); Vaníčková (2017) y Tuczak y Górzna (2012). 
A partir de la tabla 1, se desprende que todas las metodologías estudiadas están orientadas hacia cualquier tipo de organización que desee aplicar técnicas en el desarrollo de proyectos en TIC, con el fin de lograr sus objetivos y la satisfacción de los clientes, quienes se convierten en su principal foco de atención para todas.

Sí bien todas apuntan hacia los mismos resultados, sus aplicaciones a nivel de desarrollo de software para la obtención de bienes, servicios o productos se llevan a cabo de manera diferente. Es así como se puede decir que el $\mathrm{PMBOK}^{\circledR}$, por ejemplo, si bien no es una metodología como tal, se convierte en una guía que le permite a los desarrolladores confeccionar y seguir todo un plan para la elaboración de sus proyectos. Está orientado a obtener resultados a más largo plazo y su implementación debe contar con el respaldo de la alta administración si se quiere tener éxito en su aplicación. Por lo tanto, es evidente que esta metodología pareciera requerir de mayores controles y procesos en una organización, por lo cual muchos podrían calificarla de burocrática.

En contraste, SCRUM, ÁGILES y PRINCE2 ${ }^{\circledR}$, que sí son consideradas como metodologías de desarrollo en TIC, han sido creadas con el propósito de que los resultados se obtengan de una forma más rápida y sin seguir tanta estructura, como la que presenta el PMBOK ${ }^{\circledR}$. Además, están orientadas a la satisfacción del cliente y de los usuarios, son menos estructuradas y rígidas, se adaptan rápidamente a los cambios solicitados por los clientes y los aplican de manera inmediata para obtener resultados en el corto plazo. O sea, son factores positivos la versatilidad en los desarrollos y en sus aprobaciones a nivel organizacional.

Con estas tres metodologías de desarrollo, su implementación dentro de cualquier organización requiere de menos recursos humanos, ya que pueden integrar grupos de trabajo de toda la organización; es decir, «piden prestados recursos» de diferentes instancias involucradas en el desarrollo de cualquier bien, producto o servicio, para asegurar que pueden responder más rápidamente a las solicitudes y a su desarrollo. Sus plazos de entrega en los resultados son más expeditos que los que puede brindar el $\mathrm{PMBOK}^{\circledR}$, ya que este es mucho más estructurado que sus semejantes.

SCRUM y PRINCE2 ${ }^{\circledR}$ están orientadas hacia el desarrollo rápido de software, sobre todo SCRUM ${ }^{\circledR}$ que fija su atención en mantener a sus colaboradores muy motivados para obtener resultados de calidad de una forma rápida. Ambas metodologías son consideradas ágiles.

\section{Análisis de la pasantía en la UTFSM}

Las siguientes son buenas prácticas que la UTFSM lleva a cabo con el fin de fomentar cada vez más entre la comunidad universitaria, tanto a nivel administrativo, profesorado y estudiantil que aseguren acercarse al éxito en sus proyectos en donde interviene la tecnología.

1. Conformación de un comité ejecutivo integrado por representación de la parte administrativa técnica, administrativa docente y estudiantil, así como de otras instancias con poder de decisión dentro de la institución. 
2. Llevan a cabo los proyectos alineados a la estrategia organizacional y que respondan a las necesidades reales de la universidad.

3. Uso restringido de los recursos económicos, llevando a la práctica solo aquellos proyectos que le agreguen valor a la misión y visión universitaria y a su quehacer diario.

4. Se basan en la metodología de proyectos del $\mathrm{PMBOK}{ }^{\circledR}$, sobre todo en algunos procesos que llevan a cabo dentro de la universidad, pero también agregan sus propios «ingredientes» en procura de adaptarlos a las necesidades de la institución según las características de cada proyecto, así como también utilizan otras metodologías como SCRUM, ÁGILES y PRINCE2 ${ }^{\circledR}$.

4. Independencia por parte de cada departamento u oficina para presentar sus proyectos una vez cada año; sin embargo, deben de cumplir con los requisitos mínimos que han establecido para que sean revisados por el comité designado.

5. Como requisitos mínimos para que el comité revise los proyectos, estos deben contener, al menos: patrocinador, un anteproyecto que incluya una gestión de riesgos, un análisis de costos, la importancia de su ejecución y, por último, la fuente de financiamiento.

Sí bien la UTFSM no cuenta con una unidad de proyectos, sí los ejecutan de manera eficiente, procurando obtener productos de calidad con el concurso de la mayoría de los involucrados.

Tienen una ventaja competitiva, ya que por ser una institución orientada en su mayoría a la obtención de graduados en las áreas de la ingeniería e informática, cuenta con gran cantidad de recurso humano en formación, acuerpados por todo un sistema de profesores también especialistas en estas áreas, esto hace que sus proyectos tengan a la mano muchos desarrolladores dedicados a la producción de software.

Una vez realizada la revisión de literatura, abarcando las cuatro metodologías estudiadas, y al comparar las ventajas, desventajadas y facilidades en el desarrollo de software de cada una, además de haber analizado los resultados de la pasantía en la UTFSM, a continuación se presenta la propuesta de la Metodología de Proyectos TIC para la UNED.

\section{Propuesta de la Metodología de Proyectos TIC para la UNED}

Con el fin de establecer una metodología estándar en la ejecución y documentación de proyectos de TIC para la UNED, se elabora una metodología para que sea considerada por todas las personas de la institución al momento de desarrollar proyectos de esta naturaleza, esto en cumplimiento con lo establecido en las Normas Técnicas para la Gestión y el Control de las Tecnologías de Información y Comunicación de la Contraloría General de la República (CGR), de Costa Rica. 
Siguiendo las buenas prácticas en el desarrollo de una metodología de proyectos TIC, la Vicerrectoría de Planificación, en la figura de su vicerrector, se dio a la tarea de solicitar la participación de diferentes instancias, consideradas esenciales dentro de la universidad y en el desarrollo de esta metodología de proyectos en TIC, para su creación. Entre las instancias involucradas están: Dirección de Tecnología de Información y Comunicaciones (DTIC), Centro de Investigación y Evaluación Institucional, Programa de Control Interno.

Para la construcción de esta metodología, se eligió como parámetro la guía del $\mathrm{PMBOK}^{\circledR}$ debido a que se muestra como una serie de pasos cronológicos para la presentación de las solicitudes de proyectos dentro de la UNED. No obstante, cuando la instancia que los va a desarrollar determine cuál metodología utilizar internamente, podrá elegir entre las muchas que existen en el mercado. Esta fue solo una guía metodológica que ayuda a ordenar y priorizar los proyectos dentro de la universidad.

Esta metodología establece una serie de pasos para garantizar el éxito de los proyectos y que los recursos que se han de invertir para su implementación se utilicen de manera eficiente. En ella, es posible observar algunas de las consideraciones que se desprenden de la revisión de literatura, de la pasantía realizada a la UTFSM y del acuerdo de creación de la Metodología de Proyectos en TIC de la UNED, presentados en este documento. Entre ellas:

1. Todos los proyectos en TIC deben estar alineados al Plan de Desarrollo de Tecnologías de Información y Comunicación de la UNED.

2. El patrocinador de cada proyecto en TIC debe asegurar el respaldo en el desarrollo y la implementación de este.

3. Debe existir un equipo o varios equipos "multi e interdisciplinarios" de trabajo para el desarrollo de estos proyectos. Esto es menester con el fin de que las áreas usuarias e interesados, tanto internos como externos, mantengan una comunicación e interacción que garantice la pertinencia del proyecto en los contextos y poblaciones meta.

4. Todos los proyectos deben contener un apartado de riesgos que permita identificar posibles contingencias, acciones de mitigación e identificación de su nivel de impacto en la organización.

5. Se debe contar con un presupuesto que garantice el desarrollo, implementación y la ejecución del proyecto.

6. Todas las instancias de la universidad tienen la libertad de seleccionar el modelo de desarrollo que más se adecúa a las características y necesidades del proyecto.

7. Todo proyecto TIC debe contemplar un objetivo general y objetivos específicos que clarifiquen a quienes deben autorizarlos las razones de peso que sustenten su ejecución. 
8. Todo proyecto TIC debe contener al menos las seis etapas establecidas para su aprobación e implementación, a saber: anteproyecto, inicio, planeación, ejecución, control y seguimiento, evaluación y cierre.

9. Se ha establecido una serie de criterios para definir el proyecto, como los siguientes: a) Que el costo estimado de su ejecución sea de \$20000 o más, y b) Que el tiempo de implementación sea mayor o igual a tres meses de acuerdo con las actividades establecidas en un cronograma.

10. Los proyectos en TIC que cumplan con las condiciones anteriores, deben ser entregados a la Coordinación de Proyectos en TIC con el fin de verificar que cumplen con los requerimientos establecidos con anterioridad.

11. Es competencia de la Coordinación de Proyectos en TIC, una vez verificados los requisitos, trasladar a la CETIC el proyecto para su respectiva aprobación.

12. La CETIC es el único órgano autorizado para la aprobación de los proyectos en TIC.

13. Corresponde a la Coordinación en Proyectos TIC asesorar y monitorear el cumplimiento de las normas establecidas, tanto a nivel nacional e internacional, en el desarrollo y ejecución de proyectos a lo interno de la universidad.

\section{Conclusiones}

De manera independiente al tamaño y dimensión de un proyecto, la administración de toda organización debe implementar metodologías que le permitan asegurar el éxito que se propone; el caso de los proyectos relacionados con tecnologías de información y comunicación no es la excepción. Precisamente, por lo oneroso que resultan ser este tipo de proyectos, embebidos dentro del proceso y quehacer diario de la universidad, es necesario establecer controles que aseguren un mejoramiento continuo y la sostenibilidad de los sistemas que se desarrollen, así como la integridad y confiabilidad en los datos que arrojen para que la administración pueda tomar decisiones acertadas en el curso de sus actividades.

En el estudio realizado, es posible observar que entre los objetivos de la metodología de desarrollo de proyectos TIC se pueden citar:

- El control de los procesos, conocimiento de las rutas críticas, hitos, eventos, tiempos, recursos que inciden en el éxito y fracaso.

- Control de riesgos, con el fin de identificar las amenazas y las vulnerabilidades en el sistema y tomar medidas preventivas.

- Definición de alcances y limitaciones, con el objetivo de realizar propuestas realistas y factibles.

- Implementación de normas estandarizadas, reconocidas internacionalmente, que den credibilidad y validez.

- Determinar ventajas competitivas para obtener mejores resultados, que a su vez redunden en la calidad y eficiencia para hacer buen uso de los recursos. 
Partiendo de los puntos anteriormente mencionados, una Metodología de Proyectos en TIC fortalecerá la planificación estratégica de la universidad, ya que permitirá homologar las solicitudes, facultando a la administración el determinar las prioridades y necesidades alineadas con la estrategia organizacional.

Es necesario que las autoridades apoyen estas iniciativas, porque se pueden convertir en una base muy importante para la administración de los proyectos informáticos, del manejo del portafolio de proyectos, en la priorización por parte de las dependencias encargadas del desarrollo de sistemas y para el sano y responsable uso de los recursos, tanto humanos y tecnológicos, así como, por supuesto, económicos.

Por último, es posible afirmar que todas las instancias de la universidad que utilicen esta metodología estarán coadyuvando con la administración para hacer más eficientes todas las acciones que se lleven a cabo para subsanar las necesidades informáticas que se determinen y que redundarán en mejores servicios, procesos y actividades, entre otros aspectos. Esto posibilitará hacer un uso más responsable de la inversión realizada en la educación superior pública, dado que, en la medida en que se desarrollen proyectos robustos y que vengan a hacer más eficientes las actividades propias de la universidad, tanto en el área administrativa como académica, en esa misma medida se estará contribuyendo con el desarrollo de la institución y, más importante aún, del país.

\section{ABSTRACT \\ Creation of a project management methodology in Information and Communication Technologies at Universidad Estatal a Distancia}

This article summarizes the journey for the creation of a TIC project management methodology at Universidad Estatal a Distancia (UNED), which follows the requirements of the Comptroller General of the Republic (2007), which states in entry 1.5 that «the organization must manage its IT projects in such a way as to meet the objectives, requirements and terms regarding the quality, time, and budget instructions defined». To do so, first a review of literature was done, which covered PMBOK ${ }^{2}$, SCRUM, ÁGIL, PRINCE2 $®$ and an internship at Universidad Técnica Federico Santa María (UTFSM), at Chile.

The application of TIC Project management methodology allows the institutions to engage in good practices that are internationally verified.

Key words: TIC projects, development methodologies, management, risk.

RÉSUMÉ

Conception d'une méthodologie de gestion de projets en Technologies de l'information et de la Communication dans l'Université de l'État à Distance

Ce travail présente un résumé de la route suivie pour que l'Université de l'État à Distance (UNED- Universidad Estatal a Distancia) puisse avoir une méthodologie de gestion de projets dans le domaine des Technologies de l'information et de la Communication (TIC). Cette méthodologie est proposée conformément la ligne directive du Bureau Contrôleur général de la République du Costa Rica (Contraloría General de la Republica) qui établit au point 1.5 : «l'organisation doit gérer ses projets des Technologies de l'Information (TI) de sorte que les objectifs puissent être atteints, les besoins soient satisfaits en respectant la qualité, le temps ainsi que le budget, préétablis, de manière optimale». 
La conception de cette méthodologie a compris la revue de littérature des méthodologies PM$\mathrm{BOK}^{\circledR}, \mathrm{SCRUM}$, ÁGIL, PRINCE ${ }^{\circledR}$ et la réalisation d'un stage à l'Université Technique Federico Santa María (UTFSM) au Chili.

La mise en place de la méthodologie de gestion de projets TIC permet aux institutions le recours

à de bonnes pratiques, qui ont été testées au niveau international, et qui assurent la réussite.

Mots clés: Projets TIC, méthodologie de développement, gestion, risque.

\section{Fuentes consultadas}

Baham, Corey, Hirschheim, Rudy, Calderon, Andres y Kisekka, Victoria. «An Agile Methodology for the Disaster Recovery of Information Systems Under Catastrophic Scenarios». Journal Of Management Information Systems, 34, n. 3 (2017): 633-663.

Cole, Gray y Borzone, Brian. «How Agile Methodology Applies to HR Technology Solution Selection». Workforce Solutions Review, 8, n. $^{\circ} 1$ (2017): 4-6.

Ionel, Nicolás David, «Critical Analysis of the SCRUM Project Management Methodology. Annals Of The University Of Oradea», Economic Science Series, 17, n. ${ }^{\circ}$ (2008): 435-441.

Lárusdóttir, Marta Kristin, Cajander, Åsa y Gulliksen, Jan. «Informal feedback rather than performance measurements - user-centred evaluation in Scrum projects». Behaviour \& Information Technology, 33, n. ${ }^{\circ} 11$ (2014): 1118-1135.

Machado, Sampaio, Thais Cristina, Pinheiro, Plácido Rogério y Tamanini, Isabelle. «Project

management aided by verbal decision analysis approaches: a case study for the selection of the best

SCRUM practices». International Transactions In Operational Research, 22 n. ${ }^{\circ} 2$ (2015): 287-312.

Maruping, Likoebe M., Venkatesh, Viswanath y Agarwal, Ritu. «A Control Theory Perspective on Agile Methodology Use and Changing User Requirements». Information Systems Research, 20, n. 3 (2009): 377-399.

Özkan, Necmettin e Kucuk, Civian., «A Systematic Approach to Project Related Concepts of Scrum», Review Of International Comparative Management / Management Comparat International, 17, n. ${ }^{\circ} 4$ (2016): 320-334.

Project Management Institute, Inc. Guía de los Fundamentos de la Dirección de Proyectos. Pennsylvania: Newtown Square, 2004.

Tuczak, Jacek, y Górzna, Marcelina. «Ocena Skuteczności Metodyki Zarządzania Projektami PRINCE2® W Administracji Publicznej». Research Papers Of The Wroclaw University Of Economics / Prace Naukowe Uniwersytetu Ekonomicznego We Wroclawiu, 264 (2012): 282-299.

Universidad Estatal a Distancia. Plan de Mejoramiento Institucional (PMI). San José, Costa Rica, 2012.

Universidad Estatal a Distancia. Perfil del puesto Oficina de Recursos Humanos de la UNED [sin publicar], 2014.

Universidad Estatal a Distancia. Lineamientos de Política Institucional 2015-2019. Acceso: 22 de enero, 2020. http://www.uned.ac.cr/viplan/images/cppi/Lineamientos_de_Pol\%C3\%ADtica_Institucional_2015-2019. pdf

Universidad Estatal a Distancia. Lineamientos de Política Institucional 2007-2011. Acceso: 22 de enero, 2020. https://www.uned.ac.cr/conuniversitario/ images/docs_cu/politicas_generales/LineamientosPoliticaInstitucional2007-2011.pdf 
Universidad Estatal a Distancia. Plan de Desarrollo Institucional: para el fortalecimiento de la educación a distancia 2011-2015. Acceso: 22 de enero, 2020. https://www.uned.ac.cr/viplan/images/cppi/Plan_de_Desarrollo_Institucional_2011-2015.pdf

Universidad Estatal a Distancia (2017). Metodología de Proyectos en TIC. Acceso: 22 de enero, 2020. https:// www.uned.ac.cr/viplan/documentos

Universidad Estatal a Distancia (2018). Acuerdo de Mejoramiento Institucional. Acceso: 22 de enero, 2020. https://www.uned.ac.cr/ami/inicio

Universidad Técnica Federico Santa María (2015). Propuesta de Estructura Orgánica y de Planta de la Dirección de Tecnologías de Información.

Universidad Técnica Federico Santa María. «Historia». Acceso: 22 de enero, 2020. http://www.usm.cl/ universidad/historia/

Universidad Técnica Federico Santa María (2015). «Propuesta de Estructura Orgánica y de Planta de la Dirección de Tecnologías de Información». Documento de trabajo interno de la universidad.

Vaníčková, Radka. «Application of PRINCE2® Project Management Methodology». Studia Commercialia Bratislavensia, 10, n. $^{\circ} 38$ (2017): 227-238.

Walczak, Wojciech y Kuchta, Dorota. «Risks Characteristic of Agile Project Management Methodologies and Responses to Them». Operations Research \& Decisions, 23, n. ${ }^{\circ}$ (2013): 75-95. 